\title{
El síndrome de burnout en los profesionales de salud en la pandemia por la COVID-19.
}

\author{
Burnout syndrome in health care workers in the COVID-19 pandemic.
}

\section{Antonio Lozano-Vargas $1,2,3, \mathrm{a}$}

En los últimos años, el síndrome de burnout se ha convertido en un problema psicosocial significativo causado por un inadecuado manejo del estrés crónico en el ámbito laboral. El síndrome de burnout se caracteriza por la presencia de agotamiento emocional, despersonalización y la percepción de una baja realización personal. En este sentido, los profesionales de la salud son un grupo especialmente susceptible de padecer el síndrome de burnout dada la naturaleza exigente de su profesión y del entorno de trabajo (1).

Por otro lado, el síndrome de burnout puede estar asociado a la presencia de ansiedad, depresión, estrés post traumático, a una menor satisfacción profesional, a la disminución de los cuidados asistenciales y a un mayor índice de suicidio (2). En el contexto de la pandemia por la COVID-19, los profesionales de la salud que trabajan en la primera línea están expuestos a diversas situaciones de estrés; por lo cual tienen un alto riesgo de desarrollar el síndrome de burnout.

Según un estudio realizado en 100 médicos residentes en Rumania, con edades entre 24-35 años, se les aplicó la escala Maslach Burnout Inventory (MBI) dos meses después del inicio de la pandemia y se obtuvo como resultado una media de burnout de 76\% (3). Así, la prevalencia global del síndrome de burnout entre los médicos residentes en este estudio es alta, lo que demuestra que la amenaza que supone la COVID-19 es un importante factor de estrés para el personal médico (4).

En otro estudio realizado en Alemania, se aplicó las sub-escalas de depresión, ansiedad y estrés del Patient Health Questionnaire (PHQ) y la escala MBI a 75 enfermeras, 45 de ellas trabajando en primera línea contra la COVID-19 y 30 en ambientes habituales, encontrando que aquellas que trabajaban en primera línea presentaban niveles más altos de estrés, agotamiento emocional y estado de ánimo depresivo; así como niveles más bajos de realización personal en comparación con aquellas que trabajan en los ambientes habituales; las causas más comunes de burnout fueron la tensión laboral y la incertidumbre sobre el futuro (5).

Por otra parte, en otro estudio en China se aplicó la escala MBI a un total de 107 enfermeras de primera línea frente al COVID-19 (6), la edad media fue de 30,28 años y el 90,7\% eran mujeres; se observó la presencia de burnout leve en las dimensiones de agotamiento emocional y despersonalización en un 78,5\% y un $92,5 \%$ respectivamente. Sin embargo, se observó la presencia de burnout severo en la dimensión de baja realización personal en un 48,6\%; asimismo, las enfermeras con más horas de trabajo en las unidades COVID-19 presentaron un mayor agotamiento emocional y despersonalización. En relación con la edad, las enfermeras de menor edad experimentaron un mayor agotamiento emocional y una menor realización personal (6).

Sección de Psiquiatría y Salud Mental, Facultad de Medicina Alberto Hurtado, Universidad Peruana Cayetano Heredia. Lima, Perú.

2 Servicio de Neuro-Psiquiatría, Hospital Cayetano Heredia. Lima, Perú.

3 Clínica Anglo Americana. Lima, Perú.

a Médico-Psiquiatra. 
En otro estudio realizado en 880 profesionales de la salud en China se aplicó la escala de MBI y un cuestionario de estilos de afrontamiento. Los médicos fueron el 64\% y las enfermeras el 36\%. Se observó la presencia de burnout en la dimensión de agotamiento emocional en un 9,09\%, en el de despersonalización en un 50,57\%, en el de baja realización personal en un 56,59\% y un score total de 73,98\%. Por otro lado, se observó que trabajar en un área con alto riesgo de infección por la COVID-19 y tener estilos negativos de afrontamiento pueden afectar a las tres dimensiones de burnout. Asimismo, se observó que una mayor cantidad de turnos laborales nocturnos y el hecho de tener síntomas de la COVID-19 pueden aumentar el agotamiento emocional y la despersonalización, mientras que una mayor intensidad de trabajo puede aumentar el agotamiento emocional y el poseer un título profesional superior puede disminuir la realización personal (7).

Por tal motivo, es necesario atender el impacto en la salud mental de los profesionales de salud que trabajan en la primera línea contra la COVID-19, asegurar el acceso de todo el personal de salud a la atención en salud mental y brindarles un tratamiento oportuno y eficaz.

\section{REFERENCIAS BIBLIOGRÁFICAS}

1. Raudenská J, Steinerová V, Javůrková A, Urits I, Kaye AD, Viswanath $\mathrm{O}$, et al. Occupational burnout syndrome and post-traumatic stress among healthcare professionals during the novel coronavirus disease 2019 (COVID-19) pandemic. Best Pract Res Clin Anaesthesiol. 2020;34(3):553-560. doi: 10.1016/j. bpa.2020.07.008

2. Alharbi J, Jackson D, Usher K. Compassion fatigue in critical care nurses. An integrative review of the literature. Saudi Med J. 2019;40(11):1087-1097. doi: 10.15537/smj.2019.11.24569

3. Maslach C, Jackson SE, Leiter MP. Maslach burnout inventory manual. Palo Alto, CA: Consulting Psychologists Press; 1996.
4. Dimitriu MCT, Pantea-Stoian A, Smaranda AC, Nica AA, Carap AC, Constantin VD, et al. Burnout syndrome in Romanian medical residents in time of the COVID-19 pandemic. Med Hypotheses. 2020;144:109972. doi: 10.1016/j.mehy.2020.109972

5. Zerbini G, Ebigbo A, Reicherts P, Kunz M, Messman H. Psychosocial burden of healthcare professionals in times of COVID-19 - a survey conducted at the University Hospital Augsburg. Ger Med Sci. 2020;18:Doc05. doi: 10.3205/000281

6. Zhang Y, Wang C, Pan W, Zheng J, Gao J, Huang X, et al. Stress, Burnout, and Coping Strategies of Frontline Nurses During the COVID-19 Epidemic in Wuhan and Shanghai, China. Front Psychiatry. 2020;11:565520. doi: 10.3389/fpsyt.2020.565520

7. Liu X, Chen J, Wang D, Li X, Wang E, Jin Y, et al. COVID-19 Outbreak Can Change the Job Burnout in Health Care Professionals. Front Psychiatry. 2020; 11:563781. doi: 10.3389/fpsyt.2020.563781 